\title{
Hypersonic Flow around a Sphere with CLL Model of Incomplete Energy Accommodation
}

\author{
Liu, H. L., Shen, C. \\ Laboratory of High Temperature Gas Dynamics \\ Institute of Mechanics, Chinese Academy of Sciences
}

\begin{abstract}
Hypersonic rarefied flow of nitrogen over a sphere is simulated by the Direct Simulation Monte Carlo (DSMC) calculations to study the drag coefficient under Lord's diffuse scattering modelwith incomplete energy accommodation. The flow field and drag results from free-molecule flow to transition regime flow are obtained for extreme cases of complete energy accommodation and zero accommodation (diffuse elastic reflection). Available wind tunnel experimental data agree with the complete energy accommodation limit. But incomplete energy accommodation may have remarkable influence on orbital drag of a satellite. The parameters needed for this incomplete energy accommodation model are usually determined by the analysis on the result of the experiment.
\end{abstract}

\section{INTRODUCTION}

In comparison with the great attention paid to the intermolecular collision models used in simulating rarefied gas flows by the DSMC method, the effort devoted towards the accurate simulation of the molecule-surface interaction has been far from sufficient. Most DSMC calculations have been using only the simple Maxwell gas-surface interaction model that is a mixture of the diffuse and specular models. The mixture model is useful where molecular collisions in the gas phase are much more numerousthan those at the surface and the flow kinetic temperatures are not greatly different from those of the surface. But it has no sound physical foundation. Fortunately, with the wide use of computer, the remarkable improvement of its function and the great development in experiment methods and instruments, it is possible to get more accurate reflection models which are capable to compare with more reliable experiments' results. Lord ${ }^{[1]}$ has shown how the more realistic gas-surface interaction model ofCercignani and Lampis $^{[2]}$ can be incorporated into DSMC calculations (C-L-L model). The model satisfies detailed balance (reciprocity), produces physically reasonable distributions of direction and energy of re-emitted molecules and appears to be well suited to incorporation into DSMC programs. It provides a continuous spectrum of behavior from specular reflection at one end to diffuse reflection with complete energy accommodation at the other. As is well known the averaged effects of a large number of molecular impacts on the surface are discussed in terms of the tangential and normal momentum transfer coefficients, $\sigma_{t}$ and $\sigma_{n}$, respectively, and of the thermal accommodation coefficient, $\alpha$. The C-L-L model has separate accommodation coefficients $\alpha_{t}$ and $\alpha_{n}$ for the kinetic energy associated with the tangential and normal velocity components. Lord extended the model to include the case of diffuse scattering with partial energy accommodation ${ }^{[3]}$. It is observed that the C-L-L model becomes to describe more actual situation of the action between gas and body surface. This model is adopted in this paper in simulating the flow around a sphere for the two extreme cases of complete and zero energy accommodation. The flow field cases and drag coefficients among the range from free molecule flow to transition regime are calculated. Comparison with available wind tunnel data is followed by discussion.

CP585, Rarefied Gas Dynamics: $22^{\text {nd }}$ International Symposium, edited by T. J. Bartel and M. A. Gallis (C) 2001 American Institute of Physics 0-7354-0025-3/01/ $\$ 18.00$ 


\section{THE C-L-L DIFFUSE SCATTERING MODEL WITH INCOMPLETE ENERGY ACCOMMODATION}

In the C-L-L model, the transformations of the normal and tangential components of velocity of the molecule on collision with the surface are mutually independent and the scattering kernel, or probability density function of the reflected state per unit volume of phase space, therefore consists of a product of three terms, one for each component. The part of the scattering kernel for a tangential velocity component $v$ is

$$
R\left(v_{i}, v_{r}\right)=\left\{1 /[\pi \sigma(2-\sigma)]^{1 / 2}\right\} \exp -\left\{\left[v_{r}-(1-\sigma) v_{i}\right]^{2} / \sigma(2-\sigma)\right\}
$$

with a similar expression for $w$. This satisfies the reciprocity of detailed balance relation

$$
\exp -v_{i}^{2} R\left(v_{i}, v_{r}\right)=\exp -v_{r}^{2} R\left(-v_{r}, v_{i}\right)
$$

In the above equation, $v_{i}$ and $v_{r}$ are the incident and reflected velocity components measured in units of $\left(2 R T_{w}\right)^{1 / 2}$, where $T_{w}$ is the wall temperature.

The resultant magnitude of two orthogonal velocity components is used to describe the behavior of the normal velocity component. This leads to the expression

$$
R\left(u_{i}, u_{r}\right)=\frac{2 u_{r}}{\alpha_{n}} I_{0} \frac{2\left(1-\alpha_{n}\right)^{1 / 2} u_{r} u_{i}}{\alpha_{n}} \exp -\frac{u_{r}{ }^{2}+\left(1-\alpha_{n}\right) u_{i}{ }^{2}}{\alpha_{n}}
$$

where $\alpha_{n}$ is the accommodation coefficient for the kinetic energy $u^{2}$ (in units of $k T_{w}$, where $k$ is Boltzmann's constant). In the interests of consistency with this expression, the symbol $\alpha_{t}$ could be used to replace $\sigma(2-\sigma)$ in equation (1). This satisfies the reciprocity of detailed balance relation

$$
\left|u_{i}\right| \exp -u_{i}^{2} R\left(u_{i}, u_{r}\right)=u_{r} \exp -u_{r}^{2} R\left(-u_{r}, u_{i}\right)
$$

where velocities away from the wall are regarded as positive.

Through composing the scattering kernels above to the distribution of resultant speed $\left.c=\left(u^{2}+v^{2}+w^{2}\right)^{1 / 2}\right]$ of the reflected molecules, Lord constructed ${ }^{[3]}$ a scattering model that is diffuse in the aspect of direction of reflected velocity but with incomplete energy accommodation. To construct such a model one obtains a kernel that transforms the resultant speed $c_{i}$ to $c_{r}$ according to C-L-L model with identical tangential and normal energy accommodation coefficients $\alpha_{t}=\alpha_{n}=\alpha$. In general the kernel transforming the incoming velocity $\mathbf{c}_{i}\left(u_{i}, v_{i}, 0\right)$ into $\mathbf{c}_{r}\left(u_{r}, v_{r}, w_{r}\right)$ is required with $c_{i}=\sqrt{ } u_{i}^{L}+v_{i}^{L}$. But this probability is the same as the probability transforming $\mathbf{c}_{i}\left(c_{i}, 0,0\right)$ into $\mathbf{c}_{r}\left(u_{r}, v_{r}, w_{r}\right)$. The latter is more easily obtained. We consider this is a sphere polar coordinates $\mathcal{C}, \theta, \phi$ which are related with $u, v, w$ as

$$
\begin{aligned}
& u=c \cos \theta \\
& v=c \sin \theta \cos \phi \\
& w=c \sin \theta \sin \phi
\end{aligned}
$$

The required kernel $P\left(c_{i} \rightarrow c_{r}\right)$ is the product of $R\left(u_{i} \equiv c_{i}, c_{r}\right)$ (cf. (3)), $R\left(0, v_{i}\right)$ and $R\left(0, w_{i}\right)$ (cf. (1)), summed over all directions in $\theta$ and $\phi$ 


$$
\begin{aligned}
P\left(c_{i}, c_{r}\right) d c_{r} & =\int_{0}^{2 \pi} \int_{0}^{\frac{\pi}{2}} R\left(u_{i} \equiv c_{i}, u_{r}\right) R\left(0, v_{r}\right) R\left(0, w_{r}\right) c_{r}{ }^{2} \sin \theta d \theta d \phi d c_{r} \\
& =\int_{0}^{2 \pi} \int_{0}^{\frac{\pi}{2}} \frac{2 c_{r}{ }^{3} \sin \theta \cos \theta}{\pi \alpha^{2}} I_{0} \frac{2(1-\alpha)^{1 / 2}}{\alpha} c_{r} c_{i} \cos \theta \exp -\frac{c_{r}{ }^{2}+(1-\alpha) c_{i}{ }^{2}}{\alpha} d \theta d \phi d c_{r} \\
& =\frac{4 c^{3}}{\alpha^{2}} \exp -\frac{c_{r}{ }^{2}+(1-\alpha) c_{i}{ }^{2}}{\alpha} \int_{0}^{1} I_{0}\left[\frac{2(1-\alpha)^{1 / 2}}{\alpha} c_{r} c_{i} x\right] x d x d c_{r}
\end{aligned}
$$

Using the known relation for the modifiedBessel function

$$
\frac{d\left(x I_{1}(A x)\right)}{d x}=A x I_{0}(A x)
$$

equation (5) can be rewritten to

$$
\begin{aligned}
P\left(c_{i}, c_{r}\right) d c_{r} & =\frac{4 c_{r}{ }^{3}}{\alpha^{2}} \exp -\frac{c_{r}{ }^{2}+(1-\alpha) c_{i}{ }^{2}}{\alpha} \frac{\alpha}{2(1-\alpha)^{1 / 2} c_{r} c_{i}} x I_{1}\left[\frac{2(1-\alpha)^{1 / 2}}{\alpha} c_{r} c_{i} x\right]{ }_{0}^{1} d c_{r} \\
& =\frac{2 c_{r}{ }^{2} d c_{r}}{c_{i} \alpha(1-\alpha)^{1 / 2}} I_{1} \frac{2(1-\alpha)^{1 / 2} c_{r} c_{i}}{\alpha} \exp -\frac{c_{r}{ }^{2}+(1-\alpha) c_{i}{ }^{2}}{\alpha}
\end{aligned}
$$

In the case $\alpha=0$ the kernel reduces to the diffuse elastic scattering kernel, whereas $\alpha=1$ corresponds to complete accommodation.

The corresponding energy distribution is

$$
f(E) d E=\frac{E^{1 / 2} d E}{\alpha\left[(1-\alpha) E^{\prime}\right]^{1 / 2}} I_{1} \frac{2\left[(1-\alpha) E E^{\prime}\right]^{1 / 2}}{\alpha} \exp -\frac{E+(1-\alpha) E^{\prime}}{\alpha}
$$

The diffuse reflection model with incomplete energy accommodation is implemented in DSMC simulation as follows according to the above derivation. First, according to the procedure for normal velocity component (the incident value being $c_{i}$ ) the sampling of $u_{r}$ is realized, then $v_{r}$ and $w_{r}$ are obtained according to the procedure for tangential velocity components (incident values being 0 ). The value $c_{r}$ is taken as $\sqrt{ } u_{r}^{2}+v_{r}^{2}+w_{r}^{2}$. The special distribution of the direction of $c_{r}$ is realized according to even distribution of $\phi$ in $(0,2 \pi)$ and cosine distribution of $\theta$ in $(0, \pi / 2)$, that is

$$
\begin{aligned}
& \phi=2 \pi \operatorname{ranf}_{1} \\
& \cos \theta=\operatorname{ranf}_{2}
\end{aligned}
$$

where $\operatorname{ranf}_{1}$ and $\operatorname{ranf}_{2}$ are both random number uniformly distributing between 0 and 1.

The kinetic energy distribution obtained by our DSMC simulation for the test case of molecular impingement on a surface element is in excellent agreement with the theoreticalprediction.Figure 1 shows the results of Monte Carlo calculations of the energy distribution of an initiallymonoenergetic sample of 10000 molecules after diffuse reflection with incomplete energy accommodation implemented as described above from the surface for the particular values $E^{\prime}=2, \alpha=0.5$. The results are compared with calculated values of the energy distribution using equation (6) and the agreement is seen to be excellent. 


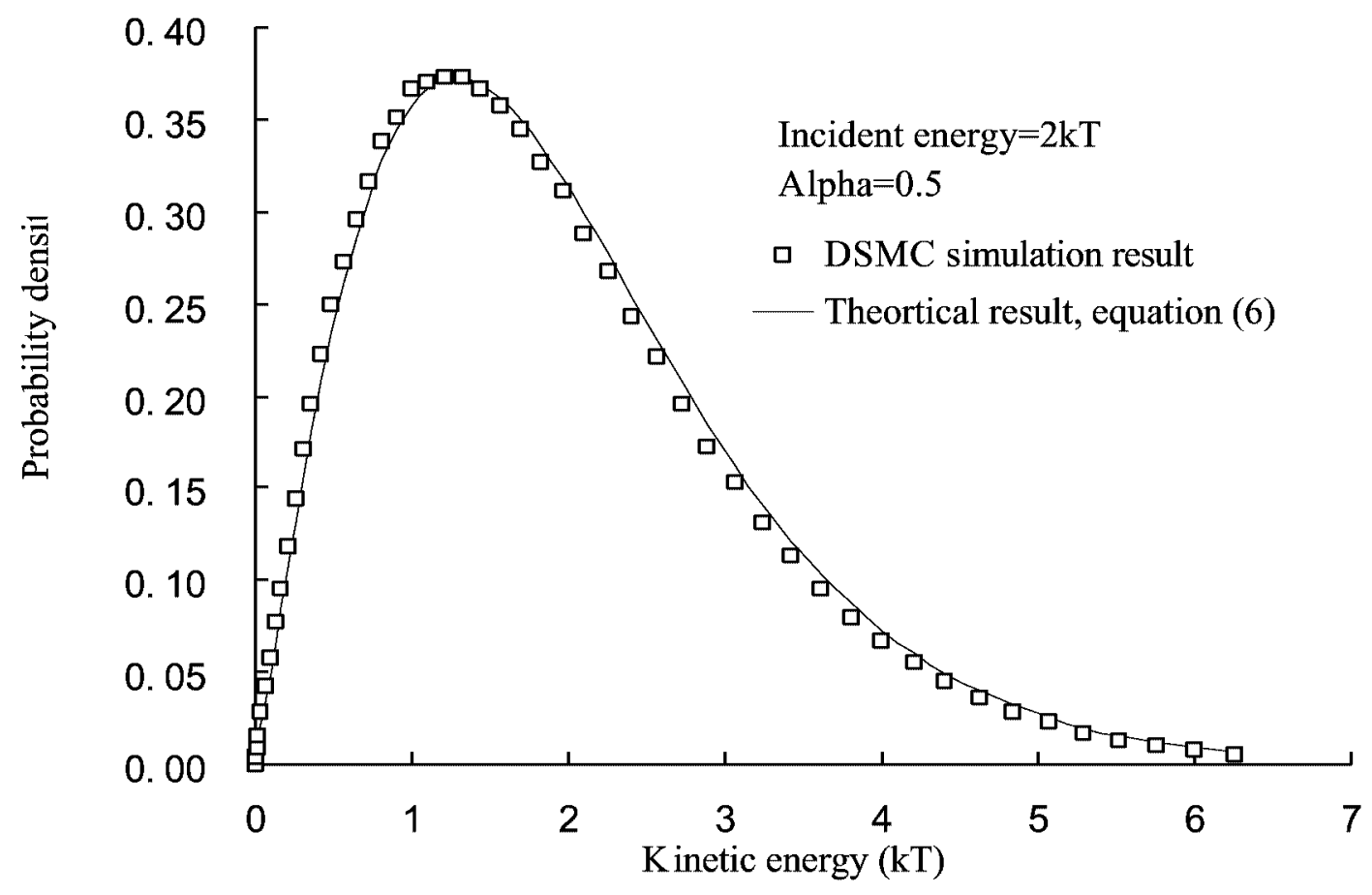

Fig. 1 Reflected energy distribution with $E^{\prime}=2$ and $\alpha=0.5$

\section{SIMULATION BY THE DSMC METHOD}

The simulation of the flow field is carried out by using the DSMC method ${ }^{4]}$. DSMC is a method proceeding from the physical simulation of the motion of large number of representative particles. The particles' positions and velocities are modified owing to their motions and collisions among them and the interactions with the boundaries. The motions and the intermolecular collisions are uncoupled and theformer are simulated deterministically and the latter are followed statistically. The method is expounded thoroughly in [2] by Bird.

A general code to implement the DSMC procedure for three-dimensional flows adapting the position element concept was developed by Shen et al. ${ }^{[5]}$, in which the body surface is described more exactly by the determination of the element surface of body configuration stretched on each surface element cube with improved accuracy in calculation of aerodynamics force and heating. In this paper, this method is adopted to simulate the flow around a sphere with a position element system of $128 \times 128 \times 128$. As the body surface is presented by the flat element surface sufficiently accurately (see [6]), it is a simple procedure to implement the C-L-L reflection model in the position element framework. In our simulation, the flow media is assumed to be adiatomic molecule gas $\mathrm{N}_{2}$. The variable hard sphere or VHS model ${ }^{[2]}$ is employed to describe the collisions between molecules.

The results of DSMC calculations of the drag coefficient for the sphere are obtained for several Knudsen numbers ranging from free-molecule to continuum flow regime with $M=8.68, T_{w} / T_{o}=0.04 \sim 0.17$. The results of two extreme cases (? for diffuse elastic reflection and? for complete energy accommodation) are given together with the cold sphere experimental data (dashed area) cited in [8] (see Fig. 3). The latter are in general dispersed near the complete energy accommodation limit (the complete diffuse reflection). The scatter of the data and the deviation 
from the complete diffuse reflection limit are explained by the difference in the ratio of the wall temperature to the stagnation temperature (varied from $0.07 \sim 0.3$ ). It is worth noting the big difference between the zero and the complete energy accommodation limits of the simulated sphere drag. In the light of exact prediction of the orbital drag of a satellite it is an unsolved problem to determine the reflection model in the environment of outer atmosphere. The strong impingement of the atmospheric molecule on the satellite surface and the outgassing caused by long exposure to the high vacuum on the orbit make the satellite surface very clean and make it behave more like a surface with incomplete energy accommodation. The present simulation shows that the incomplete accommodation has remarkable influence on orbital drag of a satellite. More work on surface-gas interaction in the laboratory and in the upper atmosphere is needed.

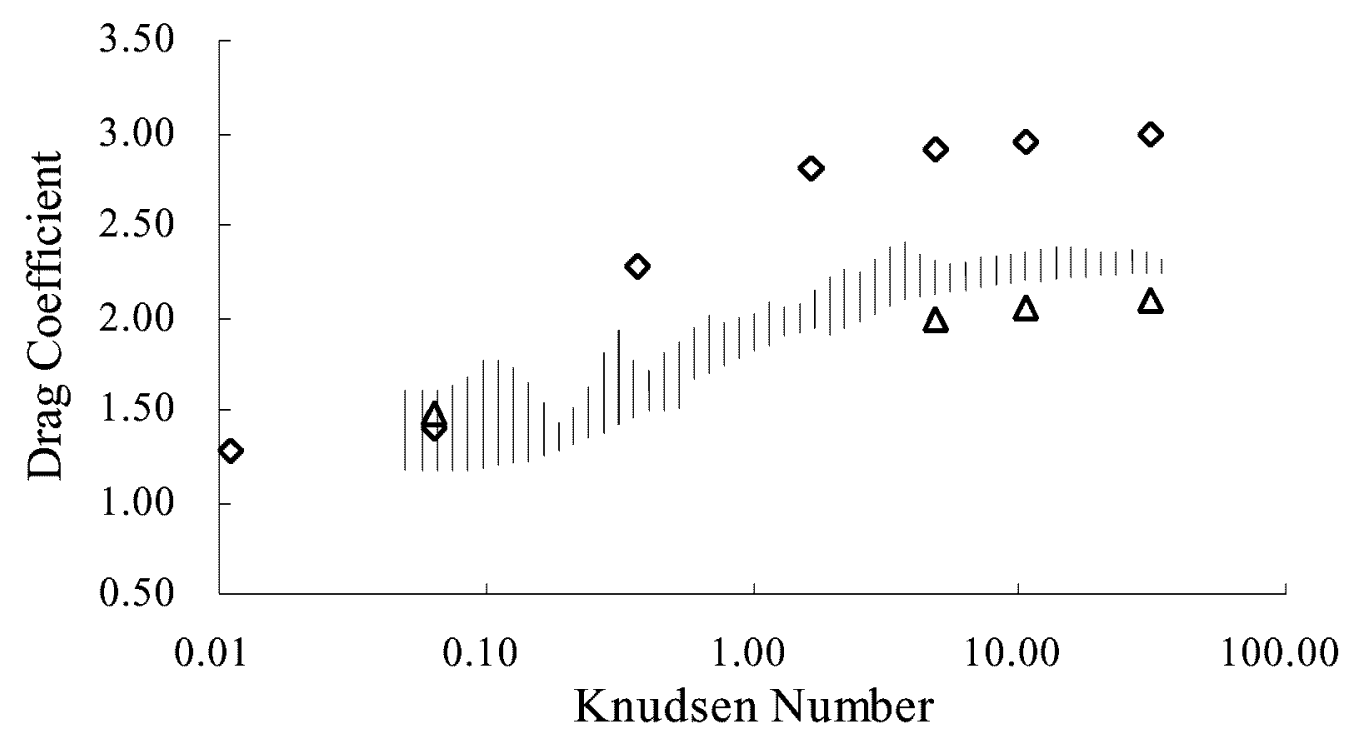

Fig.2 Sphere Drag Coefficient versus Knudsen numbe

Fig. 3 shows the density and temperature distribution along stagnation line at altitude of $130 \mathrm{~km}$ $\left(V_{\infty}=7500 \mathrm{~m} / \mathrm{s}, \rho_{\infty}=8.23 \times 10^{-9} \mathrm{~kg} / \mathrm{m}^{3}, T_{\infty}=500 \mathrm{~K}, T_{w}=350 \mathrm{~K}\right)$. From Fig. 3, one can see that on the stagnation line at position near the wall there is a good agreement of the present simulated results of the density and temperature for $\alpha=1$ with the results of our earlier calculation ${ }^{[6]}$ (not shown in the paper) and the results of Dogra et al. ${ }^{[9]}$. But as can be seen from the simulated results of density for $\alpha=0$, its values are all smaller than those values for $\alpha=1$. This phenomenon can be explained as follows: Under the condition of $\alpha=1$, the reflected velocities of the molecules emitted from the sphere surface are much smaller than the reflected velocities of those molecules under the condition of $\alpha=0$. Therefore there are more molecules near the body surface in the case $\alpha=1$ than in the case of $\alpha=0$, where the molecules with much greater magnitudes of velocity fly to farther distances. 


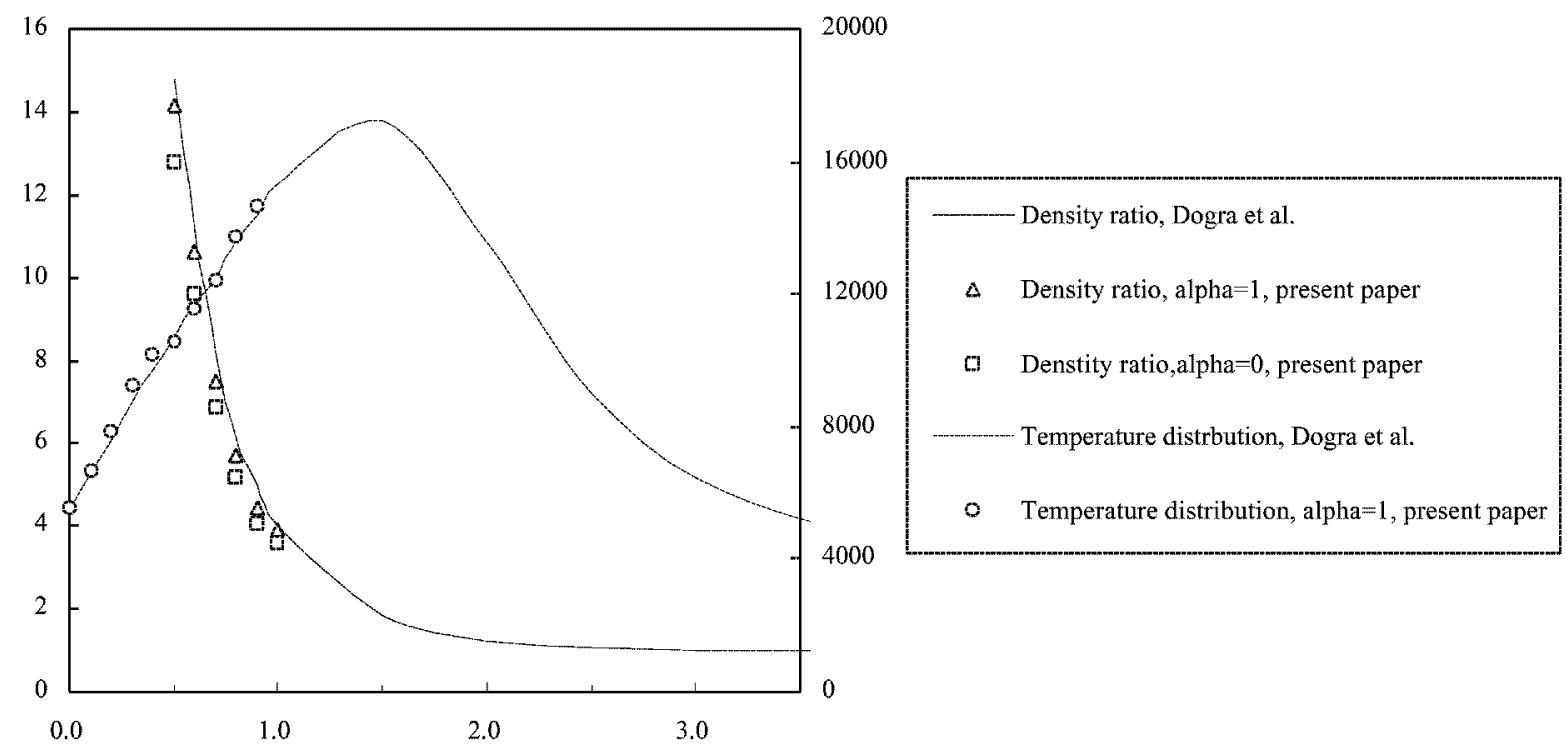

Fig. 3 Density and temperature distribution along stagnation line at altitude of $130 \mathrm{~km}$

\section{CONCLUSIONS}

Hypersonic rarefied flow of nitrogen over a sphere is simulated by the Direct Simulation Monte Carlo (DSMC) calculations to study the drag coefficient under Lord's diffuse scattering model with incomplete energy accommodation. Good agreement of available wind tunnel experimental data with the complete energy accommodation limit shows that, in present, the vast majority of drag measure is under the condition, diffuse reflection with complete energy accommodation between gas and body surface. So the assumption of diffuse reflection model is adapted at general situation.

But the obvious difference of the simulated sphere drag and the density distribution along the stagnation line between the zero and the complete energy accommodation limits should not be ignored. The incomplete energy accommodation may have remarkable influence on orbital drag of a satellite. In the light of exact prediction of the orbital drag of a satellite it is an unsolved problem to determine the reflection model in the environment of outer atmosphere.

\section{ACKNOWLEDGMENT}

The support by NNSFC (grants 19772059, 19889209) is cordially appreciated by the authors. 


\section{REFERENCES}

1. R. G. Lord, in Rarefied Gas Dynamics, ed. by A. E. Beylich, 1991, 245-252.

2. Cercignani, C., and Lampis, M., Transport Theory and Statistical Physics 1(2), 101-114, 1971.

3. R. G, Lord, Phys, Fluids A 3(4), April 1991, 706-710.

4. Bird, G A., Molecular Gas Dynamics and the Direct Simulation of Gas Flows, Clarendon Press, Oxford (1994).

5. C. Shen, J. Fan, Z. Hu, X. Xu, in Rarefied Gas Dynamics $20^{\text {th }}$, ed. by C. Shen, Peking University Press, pp. 162-167 (1997).

6. J. Fan, S. L. Peng, H. L. Liu, C. Shen, L. M. Chen, ACTA Mechanica Sinica, Vol. 31, No. 6, pp. 671-676 (1999).

7. R. G. Lord, in Rarefied Gas Dynamics, ed. by A. E. Beylich, 1991, 1427-1433.

8. K. S. Hadjimichalis, C. L. Brundin, in Rarefied Gas Dynamics, ed. by Becher \& Fiebig, vol. 2, p. D.13 (1974).

9. Dogra, V. K., Wilmoth, R. G. and Moss, J. N., Aerothermodynamics of a $1.6 \mathrm{~m}$-diameter sphere in hypersonic rarefied flow. AIAA Paper 91-0773. 